\section{INFLAMMATORY BOWEL DISEASE SECTION FREE PAPERS}

\section{OC-119 GENETIC SUSCEPTIBILITY TO CHRONIC TRICHURIS MURIS INDUCED EXPERIMENTAL COLITIS: TRANSLATION AND RELEVANCE TO HUMAN CROHN'S DISEASE}

doi:10.1136/gut.2011.239301.119

S E Levison, ${ }^{1 *}$ P Fisher, $^{2}$ W Newman, ${ }^{3} \mathrm{~J}$ McLaughlin, ${ }^{1} \mathrm{R}$ KGrencis, ${ }^{4} \mathrm{~J} \mathrm{~L}$ Pennock' ${ }^{1}$ Translational Medicine, University of Manchester, Manchester, UK; ${ }^{2}$ Computer Sciences, University of Manchester, Manchester, UK; ${ }^{3}$ Genetic Medicine, University of Manchester, Manchester, UK; ${ }^{4}$ Life Sciences, University of Manchester, Manchester, UK

Introduction Unraveling the genetic architecture of complex traits presents a true challenge. The analysis of experimental models with strict phenotypic documentation and homology to human traits, alongside sensitive scientific methodologies, will facilitate future discoveries. Trichuris muris (T muris) induces chronic colitis in susceptible mouse strains, with previously described clinical, histological, and immunological commonalities to human Crohn's disease (CD). Conversely, resistant mouse strains exhibit acute infection, with quick and full recovery. Cross-breeding susceptible and resistant mice has permitted Quantitative Trait Loci (OTL) mapping, pertaining to chronic $T$ muris inflammation.

\section{Aims}

(1.) Characterize the genetic basis of chronic Trichuris induced colitis, and identify biologically relevant susceptibility genes.

(2) Correlate initial experimental findings to human CD genome sequence variation data.

Methods An F2 inter-cross of resistant (BALB/c) and susceptible (AKR) mice were generated and infected with $T$ muris (300 ova). Colonic and systemic phenotype was established (worm burden, histology, immune response), and OTL mapping performed. Genome-wide colonic transcriptional activity of parental strains was also determined (Affymetrix). Hypothesis-free in silico work-flow analysis identified candidate genes from the OTL and expression data. Genes lacking parental strain SNPs were excluded. Polymorphic QTL genes, with differential transcription were subsequently compared to experimental and CD data for potential homology.

Results QTL associated with chronic $T$ muris colitis susceptibility were identified on 7 murine chromosomes (Chr). 144 QTL-genes displayed parental strain SNPs and significant gene expression changes in chronic colitis (ANOVA, $p<0.05$, expression fold-change $\geq \pm 1.4$ ). OTL-Chr3 demonstrated overlap with published QTL of three unrelated experimental models of colitis. Of 33 potential OTL-Chr3 gene candidates, Ptpn 22 and Fcgr1 display human and experimental homology. Additional transcribed polymorphic genes, central to biological (KEGG) pathways, were highlighted at this locus.

Conclusion 7 QTL indicate susceptibility to chronic T muris colitis. Unbiased, systematic, reductionist analysis prioritised cis-QTL gene candidates. With experimental and human trait overlap, this model and methodology can inform the biology and outcome of transmural colonic inflammation. Positional, mechanistic pathway genes may thus be identified for translational studies.

Competing interests S Levison Grant / Research Support from: MRC, P. Fisher: None Declared, W. Newman: None
Declared, J. McLaughlin: None Declared, R. Grencis: None Declared, J. Pennock: None Declared.

Keywords experimental colitis, inflammatory bowel disease, susceptibility. 